\title{
Upper bounds for regularized determinants
}

\author{
by \\ H. Gillet * and C. Soulé
}

Let $E$ be a holomorphic vector bundle on a compact Kähler manifold $X$. If we fix a metric $h$ on $E$, we get a Laplace operator $\Delta$ acting upon smooth sections of $E$ over $X$. Using the zeta function of $\Delta$, one defines its regularized determinant $\operatorname{det}^{\prime}(\Delta)$. In [5] $\S 4.1 .6$, inspired by our arithmetic Riemann-Roch theorem, we were led to conjecture that, when $h$ varies, this determinant $\operatorname{det}^{\prime}(\Delta)$ remains bounded from above.

In this paper we prove this in two special cases. The first case is when $X$ is a Riemann surface, $E$ is a line bundle and $\operatorname{dim} H^{0}(X, E)+\operatorname{dim} H^{1}(X, E) \leq 2$, and the second case is when $X=\mathbb{P}^{1}, E$ is a line bundle, and all metrics under consideration are invariant under rotation around a fixed axis. To get the desired upper bound in the first case we use an inequality of Moser and Trudinger, and its extension to arbitrary compact manifolds due to Fontana [3]. We prove the second case by direct estimates.

Though our results deal with very few cases, we find striking that inequalities as sharp as the theorem of Moser and Trudinger can be used to prove our conjecture. We hope the reader will get interested in the general question, and try to either prove or disprove our statement.

In the first paragraph we phrase the conjecture in its most general form, and give a few facts about it. Next, in the case of a line bundle over a Riemann surface, we compute the anomaly $A(\varphi)$ for the regularized determinant of the Laplace operator when a fixed metric $h_{0}$ on the line bundle is replaced by $h_{0} e^{\varphi}$. To check our theorem, we then need to bound from above the functional $A(\varphi)$ when $\varphi$ is any smooth function on the Riemann surface (resp. any function of the distance to the origin on the projective line). This is done in the next two paragraphs. At the end, we discuss the (much easier) case of the trivial line bundle on the circle, where the determinant is bounded from below.

* Supported by N.S.F grant DMS-9501500 
We thank W. Beckner, P. Chang and J. Lott for interesting discussions.

\section{Statement of the results.}

1.1. Let $X$ be a smooth, projective, equidimensional complex variety of dimension $d$ and $h_{X}$ an hermitian metric on its tangent space. The associated (normalized) Kähler form $\mu$ is defined by the formula

$$
\mu=\frac{i}{2 \pi} \sum_{\alpha, \beta} h_{X}\left(\frac{\partial}{\partial z_{\alpha}}, \frac{\partial}{\partial z_{\beta}}\right) d z_{\alpha} d \bar{z}_{\beta}
$$

where $\left(z_{\alpha}\right)$ is any local holomorphic chart on $X$.

Consider an holomorphic vector bundle $E$ on $X$, equipped with a $C^{\infty}$ hermitian metric $h$. Let $A^{0 q}(X, E), q=0, . ., d$, be the space of smooth forms of type $(0, q)$ with values in $E$. The $L^{2}$-metric on $A^{0 q}(X, E)$ is defined by the formula

$$
\langle s, t\rangle_{L^{2}}=\int_{X}\langle s(x), t(x)\rangle \mu^{d} / d !
$$

where $s, t \in A^{0 q}(X, E)$ and $\langle s(x), t(x)\rangle$ is the pointwise scalar product defined by $h$ and $h_{X}([5], \S 4.1 .1)$. The Cauchy-Riemann operator

$$
\bar{\partial}: A^{0 q}(X, E) \rightarrow A^{0, q+1}(X, E)
$$

has an adjoint $\bar{\partial}^{*}$ :

$$
\langle\bar{\partial} s, t\rangle_{L^{2}}=\left\langle s, \bar{\partial}^{*} t\right\rangle_{L^{2}}
$$

We consider the Laplace operator $\Delta_{q}=\bar{\partial}^{*} \bar{\partial}$ on $A^{0 q}(X, E)$ and its zeta function

$$
\zeta_{\Delta_{q}}(s)=\frac{1}{\Gamma(s)} \int_{0}^{\infty} \operatorname{tr}\left(e^{-\Delta_{q} t}\right) t^{s-1} \mathrm{dt}, \operatorname{Re}(s)>1 .
$$

It is known that $\zeta_{\Delta_{q}}(s)$ can be analytically continued to the whole complex plane and is regular at the origin. The regularized determinant of $\Delta_{q}$ is defined to be

$$
\operatorname{det}^{\prime}\left(\Delta_{q}\right)=\exp \left(-\zeta_{\Delta_{q}}^{\prime}(0)\right)
$$

where $\zeta_{\Delta_{q}}^{\prime}(0)$ is the value of $\frac{d}{d s} \zeta_{\Delta}(s)$ at $s=0$. Our goal is to find upper bounds for $\operatorname{det}^{\prime}\left(\Delta_{q}\right)$ when $h$ varies. 
More precisely, for any $q \geq 0$, consider the spaces

$$
B^{q}=\bar{\partial}\left(A^{0, q-1}(X, E)\right) \subset A^{0 q}(X, E), \quad q \geq 1,
$$

$B^{0}=0$, and the zeta function

$$
\zeta_{B^{q}}(s)=\operatorname{Tr}\left(\Delta_{q}^{-s} \mid B^{q}\right), \quad \operatorname{Re}(s)>d .
$$

By the Hodge decomposition theorem we have

$$
A^{0 q}(X, E)=B^{q} \oplus \bar{\partial}^{*}\left(A^{0, q+1}(X, E)\right) \oplus \operatorname{Ker}\left(\Delta_{q}\right),
$$

and the Cauchy-Riemann operator induces an isomorphism

$$
\bar{\partial}: \bar{\partial}^{*}\left(A^{0 q}(X, E)\right) \stackrel{\sim}{\longrightarrow} B^{q}
$$

such that $\bar{\partial} \Delta_{q-1}=\Delta_{q} \bar{\partial}$. It follows that

$$
\zeta_{\Delta_{q}}(s)=\zeta_{B^{q}}(s)+\zeta_{B^{q+1}}(s)
$$

hence

$$
\zeta_{B^{q+1}}(s)=\zeta_{\Delta_{q}}(s)-\zeta_{\Delta_{q-1}}(s)+\zeta_{\Delta_{q-2}}(s)+\cdots+(-1)^{q} \zeta_{\Delta_{0}}(s) .
$$

This implies that $\zeta_{B^{q}}(s)$ converges when $\operatorname{Re}(s)>d$, has a meromorphic continuation to the whole complex plane, and is regular at the origin. Define

$$
D_{q}(E, h)=\exp \left(-\zeta_{B^{q}}^{\prime}(0)\right) .
$$

In [5], §4.1.6, we proposed the following

Conjecture. There exists a constant $C_{q}(E)$ such that, for any choice of a metric $h$ on $E$,

$$
D_{q}(E, h) \leq C_{q}(E) .
$$

\subsection{Remarks on the conjecture}

1.2.1. In the conjecture above both $D_{q}(E, h)$ and $C_{q}(E)$ depend in general on the metric $h_{X}$ on $X$. 
Notice that, for any real constant $t>0$,

$$
D_{q}(E, t h)=D_{q}(E, h) .
$$

Indeed, when $h$ is replaced by $t h$, the $L^{2}$-metric on $A^{0 q}(X, E)$ gets multiplied by the same factor $t$ for all $q \geq 0$, therefore $\Delta_{q}$ remains unchanged.

Furthermore, if $E^{\vee}=E^{*} \otimes \Lambda^{d}\left(T X^{*}\right)$ is Poincaré dual of $E$ and $h^{\vee}$ the metric on $E^{\vee}$ induced by $h$ and $h_{X}$, the Poincaré-Serre duality implies that

$$
D_{q}(E, h)=D_{d+1-q}\left(E^{\vee}, h^{\vee}\right) .
$$

Therefore the conjecture is stable under scaling and duality.

1.2.2. Our inspiration to make this conjecture was number theoretic. Assume that $X$ is the set $\mathcal{X}(\mathbb{C})$ of complex points of a regular projective flat scheme $\mathcal{X}$ over $\mathbb{Z}$ and that $E$ is the holomorphic vector bundle defined by an algebraic vector bundle $\mathcal{E}$ on $\mathcal{X}$. In loc.cit. we defined arithmetic Betti numbers

$$
b_{q}(\mathcal{E}, h) \in \mathbb{R}, \quad 0 \leq q \leq d+1,
$$

as follows . When $M$ is a finitely generated abelian group, equipped with a norm $\|\cdot\|$ on its real span $M \underset{\mathbb{Z}}{\otimes} \mathbb{R}$, we let

$$
h^{0}(M,\|\cdot\|)=\log \#\{m \in M /\|m\| \leq 1\}
$$

and

$$
h^{1}(M,\|\cdot\|)=h^{0}\left(M^{*},\|\cdot\|^{*}\right) .
$$

We consider the coherent cohomology groups $H^{q}(\mathcal{X}, \mathcal{E}), q \geq 0$, equipped with their $L^{2}$ metric. Then we let

$$
b_{q}(\mathcal{E}, h)=h^{0}\left(H^{q}(\mathcal{X}, \mathcal{E})\right)+h^{1}\left(H^{q-1}(\mathcal{X}, \mathcal{E})\right)+\frac{1}{2} \zeta_{B^{q}}^{\prime}(0) .
$$

In [5], loc.cit., we gave properties of these numbers (duality, Euler characteristic formula) which partially justified calling them Betti numbers. However, a basic property should be that each $b_{q}(\mathcal{E}, h)$ is nonnegative, or at least bounded below. This led us to the conjecture in $\S 1.1$.

1.2.3. It would be of interest to find some interpretation of our conjecture in mathematical physics. In [6], the Moser-Trudinger inequality (see $\S 2.3$ below) is 
interpreted as the existence of a lower bound for a free energy functional, and it is derived in op.cit. Prop. 4 from the Gibbs variational principle.

1.2.4. A stronger version of the conjecture consists in requiring that $C_{q}(E)$ depends only on the $C^{\infty}$ bundle underlying $E$, and not on its holomorphic structure. Results like [10] Proposition 3 (due to Miyaoka and based on a result of Selberg, [10] Theorem 4), which says that when $d=1$ and when $E$ is a flat unitary bundle, the following holds

$$
\log D_{1}(E, h) \leq \text { constant } \cdot \operatorname{rank}(E)
$$

points in this direction.

\subsection{Results}

From now on we assume that $X$ is a curve $(d=1)$ and that $E$ is a line bundle $L$. We then take $q=1$ and we write $D(L, h)$ instead of $D_{1}(E, h)=\operatorname{det}^{\prime}\left(\Delta_{0}\right)$.

\section{Theorem 1.}

i) If

$$
\operatorname{dim} H^{0}(X, L)+\operatorname{dim} H^{1}(X, L) \leq 2,
$$

there exists a constant $C(L)$ such that, for any metric $h$ on $L$,

$$
D(L, h) \leq C(L)
$$

ii) Assume that $X=\mathbb{P}^{1}(\mathbb{C})$ is the complex projective line, that $h_{X}$ is invariant under rotation, and that $L=\mathcal{O}(n), n \in \mathbb{Z}$. Then there is a constant $C(n)$ such that, for any metric $h$ on $L$ invariant under rotation,

$$
D(L, h) \leq C(n)
$$

To clarify statement ii) above, let us write $z=r e^{i \theta}$ the standard coordinate on $\mathbb{C} \subset \mathbb{P}^{1}(\mathbb{C})$. Given any $\alpha$, we let $r_{\alpha}(z)=e^{i \alpha} z$ be the rotation of angle $\alpha$. A metric $h_{X}$ on $X$ (resp. $h$ on $L$ ) is said to be invariant under rotation when $r_{\alpha}^{*}\left(h_{X}\right)=h_{X}\left(\right.$ resp. $\left.r_{\alpha}^{*}(h)=h\right)$ for all values of $\alpha$.

\section{An anomaly formula.}


2.1. We fix $X, h_{X}$ and $L$ as in $\S 1.3$. Let $h_{0}$ and $h=h_{0} \exp (\varphi)$ be two hermitian metrics on $L$, with $\varphi$ a smooth real valued function on $X$. We shall give a formula comparing the determinants $D(L, h)$ and $D\left(L, h_{0}\right)$.

Let $b_{0}=\operatorname{dim} H^{0}(X, L)$ and $b_{1}=\operatorname{dim} H^{1}(X, L)$. We endow $H^{0}(X, L)=$ $\operatorname{ker}(\bar{\partial}) \subset A^{00}(X, L)$ and $H^{1}(X, L)=\operatorname{ker}\left(\bar{\partial}^{*}\right) \subset A^{01}(X, L)$ with the $L^{2}$-metric coming from $h_{0}$. Let $\left(\alpha_{i}\right), i=1, \ldots, b_{0}$, be an orthonormal basis of $H^{0}(X, L)$, and $\left(\beta_{i}\right), i=1, \ldots, b_{1}$, an orthonormal basis of $H^{1}(X, L)$. If $1 \leq i, j \leq b_{0}$ we let $\left\langle\alpha_{i}, \alpha_{j}\right\rangle$ be the pointwise scalar product of $\alpha_{i}$ with $\alpha_{j}$. We define similarly $\left\langle\beta_{i}, \beta_{j}\right\rangle$, $1 \leq i, j \leq b_{1}$.

If $d=\partial+\bar{\partial}$ we let $d^{c}=\frac{\partial-\bar{\partial}}{4 \pi i}$, so that $d d^{c}=\frac{\bar{\partial} \partial}{2 \pi i}$.

Denote by $c_{1}\left(T_{X}, h_{X}\right)$ the first Chern form of the tangent bundle to $X$, and by $c_{1}\left(L, h_{0}\right)$ the first Chern form of $\left(L, h_{0}\right)$. Clearly, to prove Theorem 1 it will be enough to show that, under the given hypotheses, the quantity

$$
A(\varphi)=\log D(L, h)-\log D\left(L, h_{0}\right)
$$

remains bounded from above when $\varphi$ varies.

Proposition 1. The following formula holds:

$$
\begin{aligned}
& A(\varphi) \\
= & \frac{1}{2} \int_{X} \varphi d d^{c}(\varphi)-\int_{X} \varphi\left(c_{1}\left(T_{X}, h_{X}\right)+c_{1}\left(L, h_{0}\right)\right) \\
+ & \log \operatorname{det}\left(\int_{X} e^{\varphi}\left\langle\alpha_{i}, \alpha_{j}\right\rangle \mu\right)_{1 \leq i, j \leq b_{0}} \\
+ & \log \operatorname{det}\left(\int_{X} e^{-\varphi}\left\langle\beta_{i}, \beta_{j}\right\rangle \mu\right)_{1 \leq i, j \leq b_{1}}
\end{aligned}
$$

2.2. To prove Proposition 1 we consider the Quillen metric [9] on the complex line

$$
\lambda(L)=\Lambda^{b_{0}} H^{0}(X, L) \otimes\left(\Lambda^{b_{1}} H^{0}(X, L)\right)^{*} .
$$

It is defined as the quotient of the $L^{2}$-metric by the determinant of the Laplace operator:

$$
\|\cdot\|_{Q, h}^{2}=\|\cdot\|_{L^{2}, h}^{2} D(L, h)^{-1} .
$$


Therefore we get

$$
A(\varphi)=\log \frac{\|\cdot\|_{L^{2}, h}^{2}}{\|\cdot\|_{L^{2}, h_{0}}^{2}}-\log \frac{\|\cdot\|_{Q, h}^{2}}{\|\cdot\|_{Q, h_{0}}^{2}},
$$

and we are led to compute the variation of both the $L^{2}$-metric and the Quillen metric on $\lambda(L)$.

Concerning the Quillen metric, we can use the anomaly formula in [1], Theorem 0.3. Let $\widetilde{c h}\left(h, h_{0}\right)$ be the Bott-Chern secondary characteristic class of $L$, which satisfies

$$
d d^{c} \widetilde{\operatorname{ch}}\left(h, h_{0}\right)=\operatorname{ch}(L, h)-\operatorname{ch}\left(L, h_{0}\right)
$$

where $\operatorname{ch}(L, h)=\exp \left(c_{1}(L, h)\right)$ is the Chern character form of $(L, h)$. If $\operatorname{Td}\left(T_{X}\right.$, $\left.h_{X}\right)$ is the form representing the Todd class of $X$ defined using $h_{X}$, the following holds (loc.cit.):

$$
-\log \frac{\|\cdot\|_{Q, h}^{2}}{\|\cdot\|_{Q, h_{0}}^{2}}=\int_{X} \widetilde{\operatorname{ch}}\left(h, h_{0}\right) \operatorname{Td}\left(T_{X}, h_{X}\right) .
$$

Since $X$ has dimension one, we have

$$
\begin{aligned}
& \int_{X} \tilde{\operatorname{ch}}\left(h, h_{0}\right) \operatorname{Td}\left(T_{X}, h_{X}\right) \\
= & \int_{X} \widetilde{c h}_{2}\left(h, h_{0}\right)+\int_{X} \widetilde{c h}_{1}\left(h, h_{0}\right) c_{1}\left(T_{X}, h_{X}\right),
\end{aligned}
$$

where $\widetilde{c h}_{p}$ is the component of degree $(p-1, p-1)$ of $\widetilde{c h}$.

Let $\left(\mathcal{O}_{X}, e^{\varphi}\right)$ be the trivial line bundle equipped with the metric such that $\|1\|^{2}=\exp (\varphi)$. Since

$$
(L, h)=\left(L, h_{0}\right) \otimes\left(\mathcal{O}_{X}, e^{\varphi}\right)
$$

we deduce from [4], Proposition 1.3.3 (and formula (1.3.5.2)) that

$$
\widetilde{\operatorname{ch}}\left(h, h_{0}\right)=\operatorname{ch}\left(L, h_{0}\right) \widetilde{c h}\left(e^{\varphi}, 1\right)
$$

To compute the Bott-Chern class ch $\left(e^{\varphi}, 1\right)$ comparing the metric such that $\|1\|^{2}=$ $\exp (\varphi)$ with the trivial metric on $\mathcal{O}_{X}$, first notice that

$$
\widetilde{c h}_{1}\left(e^{\varphi}, 1\right)=\widetilde{c}_{1}\left(e^{\varphi}, 1\right)=-\varphi
$$


by [4], (1.2.5.1). Furthermore, from [4] (1.3.1.2) we get

$$
\begin{gathered}
\widetilde{c h}_{2}\left(e^{\varphi}, 1\right)=\frac{1}{2} \widetilde{c}_{1}^{2}\left(e^{\varphi}, 1\right) \\
=\frac{1}{2} c_{1}\left(e^{\varphi}\right) \widetilde{c}_{1}\left(e^{\varphi}, 1\right)=\frac{1}{2} d d^{c}(\varphi) \varphi .
\end{gathered}
$$

So we conclude that

(6) $-\log \frac{\|\cdot\|_{Q, h}^{2}}{\|\cdot\|_{Q, h_{0}}^{2}}=-\int_{X} c_{1}\left(L, h_{0}\right) \varphi+\frac{1}{2} \int_{X} \varphi d d^{c}(\varphi)-\int_{X} \varphi c_{1}\left(T_{X}, h_{X}\right)$.

2.3. Now we have to compute the variation of the $L^{2}$-norm on $\lambda(L)$. Since $\left(\alpha_{i}\right)$ is an orthonormal basis of $\operatorname{Ker}(\bar{\partial})$ for the $L^{2}$-metric defined by $h_{0}$, the change of metric on $\Lambda^{b_{0}} H^{0}(X, L)$ is the determinant

$$
\begin{aligned}
q_{0} & =\operatorname{det}\left(\left\langle\alpha_{i}, \alpha_{j}\right\rangle_{L^{2}, h}\right) \\
& =\operatorname{det}\left(\int_{X} e^{\varphi}\left\langle\alpha_{i}, \alpha_{j}\right\rangle \mu\right) .
\end{aligned}
$$

For $H^{1}$ the situation is more complicated since the kernel of $\bar{\partial}^{*}$ changes when $h_{0}$ is replaced by $h$. If $\psi_{i} \in A^{01}(X, L)$ is the harmonic form for the metric coming from $h$ which is cohomologous to $\beta_{i}, 1 \leq i \leq b_{1}$, the change of metric on $\Lambda^{b_{1}} H^{1}(X, L)$ is

$$
q_{1}=\operatorname{det}\left(\left\langle\psi_{i}, \psi_{j}\right\rangle_{L^{2}, h}\right)
$$

Note that $\bar{\partial}_{h}^{*}\left(e^{-\varphi} \beta_{i}\right)=0$ since, for any section $s \in A^{00}(L)$,

$$
\int_{X}\left\langle\bar{\partial} s, e^{-\varphi} \beta_{i}\right\rangle_{h} \mu=\int_{X}\left\langle\bar{\partial} s, \beta_{i}\right\rangle \mu=0 .
$$

Therefore there are complex numbers $a_{i j}, 1 \leq i, j \leq b_{1}$, such that

$$
e^{-\varphi} \beta_{i}=\sum_{j=1}^{b_{1}} a_{i j} \psi_{j}
$$

It follows that

$$
\operatorname{det}\left(\left\langle\psi_{i}, \psi_{j}\right\rangle_{L^{2}, h}\right)=\operatorname{det}\left(\left\langle e^{-\varphi} \beta_{i}, e^{-\varphi} \beta_{j}\right\rangle_{L^{2}, h}\right) \operatorname{det}\left(a_{i j}\right)^{-2}
$$


Note that

$$
\left\langle e^{-\varphi} \beta_{i}, e^{-\varphi} \beta_{j}\right\rangle_{L^{2}, h}=\int_{X} e^{-\varphi}\left\langle\beta_{i}, \beta_{j}\right\rangle \mu
$$

To compute $\left(a_{i j}\right)$ let us introduce $\gamma_{i}=* \beta_{i}, 1 \leq i \leq b_{1}$, where $*$ is the star operator defined by $h_{X}$ and $h_{0}$. Then $\gamma_{i}$ is a smooth section of the Serre dual $\Omega_{X}^{1} \otimes L^{*}$ of $L$ such that $\bar{\partial}\left(\gamma_{i}\right)=0\left(\right.$ since $\left.\bar{\partial}_{h_{0}}^{*}\left(\beta_{i}\right)=0\right)$ and

$$
\left\langle\beta_{i}, \beta_{j}\right\rangle_{h_{0}} \mu=\beta_{i} \gamma_{j} \quad, \quad 1 \leq i, j \leq b_{1}
$$

Since $\left(\beta_{i}\right)$ is orthonormal and $\psi_{i}$ is cohomologous to $\beta_{i}$ we get

$$
\int_{X} \psi_{i} \gamma_{j}=\int_{X} \beta_{i} \gamma_{j}=\delta_{i j}
$$

Using (9) we deduce that

$$
\int_{X} e^{-\varphi} \beta_{i} \gamma_{k}=\sum_{j=1}^{b_{1}} a_{i j} \int_{X} \psi_{j} \gamma_{k}=a_{i k}
$$

In other words

$$
a_{i k}=\int_{X} e^{-\varphi}\left\langle\beta_{i}, \beta_{k}\right\rangle_{h_{0}} \mu
$$

From (8), (10), (11), (12) we conclude that

$$
q_{1}=\operatorname{det}\left(\int_{X} e^{-\varphi}\left\langle\beta_{i}, \beta_{j}\right\rangle_{h_{0}} \mu\right)^{-1} .
$$

From (7) and (13) we get

$$
\begin{gathered}
\log \frac{\|\cdot\|_{L^{2}, h}^{2}}{\|\cdot\|_{L^{2}, h_{0}}^{2}}=\log \left(q_{0}\right)-\log \left(q_{1}\right) \\
=\log \operatorname{det}\left(\int_{X} e^{\varphi}\left\langle\alpha_{i}, \alpha_{j}\right\rangle \mu\right)+\log \operatorname{det}\left(\int_{X} e^{-\varphi}\left\langle\beta_{i}, \beta_{j}\right\rangle \mu\right) .
\end{gathered}
$$

Proposition 1 follows from (5), (6) and (14).

2.4. Corollary 1. Under the assumption of Proposition 1, if $b_{1}=0$ and if the conjecture holds for one choice of metric $h_{X}$ on $X$, it holds for any other choice of metric on $X$. 
Proof. Assume $h_{X}$ gets replaced by $e^{\rho} h_{X}$, where $\rho \in C^{\infty}(X)$. Then $\mu$ is replaced by $\mu^{\prime}=e^{\rho} \mu$. Let $\left(\alpha_{i}^{\prime}\right)$ be an orthonormal basis of $H^{0}(X, L)$ for the $L^{2}$ metric defined by $h_{X}^{\prime}$ and $h_{0}$. We may write

$$
\alpha_{i}^{\prime}=\sum_{j=1}^{b_{0}} a_{i j} \alpha_{j},
$$

$1 \leq i \leq b_{0}$, where the square matrix $M=\left(a_{i j}\right)$ is independent of $\varphi$. Therefore $A(\varphi)$ is replaced by

$$
\begin{aligned}
B(\varphi) & =\frac{1}{2} \int_{X} \varphi d d^{c}(\varphi)-\int_{X} \varphi\left(c_{1}\left(T_{X}, h_{X}\right)-d d^{c} \rho+c_{1}\left(L, h_{0}\right)\right) \\
& +\log \operatorname{det}\left(\int_{X} e^{\varphi+\rho}\left\langle\alpha_{i}, \alpha_{j}\right\rangle \mu\right)_{1 \leq i, j \leq b_{0}} \\
& +2 \log |\operatorname{det}(M)| .
\end{aligned}
$$

If we let $\psi=\varphi+\rho$, since

$$
2 \int_{X} \varphi d d^{c}(\rho)=\int_{X}\left(\varphi d d^{c} \rho+\rho d d^{c} \varphi\right)
$$

we get

$$
B(\varphi)=A(\psi)-\frac{1}{2} \int_{X} \rho d d^{c}(\rho)+2 \log |\operatorname{det}(M)| .
$$

When $\rho$ is chosen, if $A(\psi)$ is bounded, so is $B(\varphi)$.

q.e.d.

\section{Proof of Theorem 1 in case i).}

First notice that, because of (1), we can impose the condition

$$
\int_{X} \varphi \mu=0 .
$$

On the other hand, we can choose the reference metric $h_{0}$ in such a way that the form $c_{1}\left(T_{X}, h_{X}\right)+c_{1}\left(C, h_{0}\right)$ is proportional to $\mu$. Together with (15), this implies that the summand

$$
\int_{X} \varphi\left(c_{1}\left(T_{X}, h_{X}\right)+c_{1}\left(L, h_{0}\right)\right)
$$

in Proposition 1 vanishes. 
Now let $A$ be an upper bound on $X$ for the $C^{\infty}$ functions $\left|\left\langle\alpha_{i}, \alpha_{j}\right\rangle\right|, 1 \leq i, j \leq$ $b_{0}$, and $\left|\left\langle\beta_{i}, \beta_{j}\right\rangle\right|, 1 \leq i, j \leq b_{1}$. We get

$$
\log \operatorname{det}\left(\int_{X} e^{\varphi}\left\langle\alpha_{i}, \alpha_{j}\right\rangle \mu\right) \leq b_{0} \log \int_{X} e^{\varphi} \mu+b_{0} \log (A)+\log \left(b_{0} !\right)
$$

and

$$
\log \operatorname{det}\left(\int_{X} e^{-\varphi}\left\langle\beta_{i}, \beta_{j}\right\rangle \mu\right) \leq b_{1} \log \int_{X} e^{-\varphi} \mu+b_{1} \log (A)+\log \left(b_{1} !\right) .
$$

So Proposition 1 implies

$$
A(\varphi) \leq \frac{1}{2} \int_{X} \varphi d d^{c} \varphi+b_{0} \log \int_{X} e^{\varphi} \mu+b_{1} \log \int_{X} e^{-\varphi} \mu+c_{1},
$$

for some constant $c_{1} \geq 0$ independent of $\varphi$.

Let $\nabla$ be the gradient defined by $h_{X}$. A local computation shows that

$$
\int_{X} \varphi d d^{c}(\varphi)=-\frac{1}{4 \pi} \int_{X}|\nabla \varphi|^{2} \mu .
$$

We use now an inequality due to Fontana [3], Theorem 1.7, which extends to arbitrary compact manifolds a result of Moser and Trudinger for the sphere and open domains in $\mathbb{R}^{n}$ [7]. Namely, given any smooth real function $f$ on $X$ such that

$$
\int_{X}|\nabla f|^{2} \mu \leq 1 \text { and } \int_{X} f \mu=0,
$$

we have

$$
\log \int_{X} \exp \left(4 \pi f^{2}\right) \mu \leq c_{2},
$$

where $c_{2}$ is a constant which does not depend on $f$. From this inequality it follows that, for any smooth real function $g$ on $X$ such that $\int_{X} g \mu=0$,

$$
\log \int_{X} \exp (g) \mu \leq c_{2}+\frac{1}{16 \pi} \int_{X}|\nabla g|^{2} \mu .
$$

Indeed, if we let $B=\int_{X}|\nabla g|^{2} \mu$ and $f=g B^{-1 / 2}$, we have

$$
4 \pi f^{2}-g+\frac{B}{16 \pi}=4 \pi\left(f-\frac{\sqrt{B}}{8 \pi}\right)^{2} \geq 0 .
$$


Therefore (18) gives

$$
\log \int_{X} \exp (g) \mu \leq \log \int_{X} \exp \left(4 \pi f^{2}\right) \mu+\frac{B}{16 \pi} \leq c_{2}+\frac{1}{16 \pi} \int_{X}|\nabla g|^{2} \mu .
$$

If we apply the inequality (19) to $\varphi$ and $-\varphi$ we get, from (16) and (17), the inequality

$$
A(\varphi) \leq-\frac{1}{8 \pi} \int_{X}|\nabla \varphi|^{2} \mu+\frac{b_{0}}{16 \pi} \int_{X}|\nabla \varphi|^{2} \mu+\frac{b_{1}}{16 \pi} \int_{X}|\nabla \varphi|^{2} \mu+c_{1}+2 c_{2} .
$$

When $b_{0}+b_{1} \leq 2$ we conclude that

$$
A(\varphi) \leq c_{1}+2 c_{2}
$$

q.e.d.

\section{Proof of Theorem 1 in case ii).}

4.1. We assume that $X=\mathbb{P}^{1}$ is the complex projective line and that $L=\mathcal{O}(n)$, $n \geq 1$. Then $b_{1}=0$ and $b_{0}=n+1$. Furthermore

$$
H^{0}(X, \mathcal{O}(n))=S^{n} H^{0}(X, \mathcal{O}(1))
$$

is the space of homogeneous polynomials of degree $n$ in two variables. Consider the canonical exact sequence of sheaves

$$
0 \rightarrow \mathcal{O}(-1) \rightarrow \mathbb{C}^{2} \rightarrow \mathcal{O}(1) \rightarrow 0
$$

and denote by $A, B \in H^{0}(X, \mathcal{O}(1))$ the images of the vectors $(1,0),(0,1)$ in $\mathbb{C}^{2}=H^{0}\left(X, \mathbb{C}^{2}\right)$. Choose on $\mathcal{O}(1)$ the metric $h_{0}$ induced by the standard metric on $\mathbb{C}^{2}$. At a point $P$ with homogeneous coordinates $(u, v)$ in $\mathbb{P}^{1}$ the lift of $A$ to $\mathbb{C}^{2}$ which is orthogonal to the vector $(u, v) \in \mathcal{O}(-1)_{P}$ is given by

$$
A^{\perp}=\left(|v|^{2},-\bar{u} v\right)\left(|u|^{2}+|v|^{2}\right)^{-1} .
$$

Similarly $B$ lifts to

$$
B^{\perp}=\left(-u \bar{v},|u|^{2}\right)\left(|u|^{2}+|v|^{2}\right)^{-1} .
$$

If $P$ lies on the affine line $\mathbb{A}^{1} \subset \mathbb{P}^{1}$ with affine coordinate $z$, these vectors become

$$
A^{\perp}=(1,-\bar{z}) N^{-1}
$$


and

$$
B^{\perp}=\left(-z,|z|^{2}\right) N^{-1}
$$

where $N=|z|^{2}+1$. The scalar products of the sections $A$ and $B$ of $H^{0}(X, \mathcal{O}(1))$ at the point $P$ are thus given by

$$
\begin{gathered}
\langle A, A\rangle=A^{\perp} \cdot A^{\perp}=N^{-1}, \\
\langle A, B\rangle=A^{\perp} \cdot B^{\perp}=-\bar{z} N^{-1},
\end{gathered}
$$

and

$$
\langle B, B\rangle=B^{\perp} \cdot B^{\perp}=|z|^{2} N^{-1}
$$

An orthogonal basis of $H^{0}(X, \mathcal{O}(n))$ is the set of monomials $\left(A^{i} B^{j}, i+j=n\right)$, where $A^{i} B^{j}$ is the symmetrization of the vector $A^{\otimes i} \otimes B^{\otimes j}$ in $H^{0}(X, \mathcal{O}(1))^{\otimes n}$. Using (20) we see that the standard metric on $S^{n} H^{0}(X, \mathcal{O}(1))$, which is (a constant multiple of) the $L^{2}$-metric on $H^{0}(X, \mathcal{O}(n))$, is such that

$$
\left\langle A^{i} B^{j}, A^{k} B^{\ell}\right\rangle=\left(\begin{array}{c}
n \\
j
\end{array}\right)\left(\begin{array}{l}
n \\
\ell
\end{array}\right) \frac{(-z)^{j}(-\bar{z})^{\ell}}{N^{n}}
$$

4.2. To prove Theorem 1 ii), we may assume $n>0$, because of (2). By the argument of Corollary 1, we may also assume that both $h_{0}$ and $h_{X}$ are the standard metrics. In particular

$$
\mu=\frac{d z d \bar{z}}{2 i \pi\left(1+|z|^{2}\right)^{2}}
$$

and $c_{1}\left(L, h_{0}\right)$ is a multiple of $\mu$. By 1.2. a) we may finally assume that

$$
\int_{X} \varphi \mu=0
$$

From the previous section, an orthonormal basis $\left(\alpha_{i}\right)$ of $H^{0}(X, L)$ is given by the elements

$$
A^{k} B^{\ell} /\left\|A^{k} B^{\ell}\right\|_{L^{2}}, \quad k+\ell=n .
$$

From (21), since $\varphi$ and $\mu$ are invariant under rotation, we conclude that

$$
\int_{X} e^{\varphi}\left\langle\alpha_{i}, \alpha_{j}\right\rangle \mu=0
$$


when $i \neq j$. From Proposition 1 we get

$$
\begin{aligned}
A(\varphi) & =\frac{1}{2} \int_{X} \varphi d d^{c}(\varphi) \\
& +\sum_{i=0}^{n} \log \int_{X} e^{\varphi} \frac{|z|^{2 i}}{\left(1+|z|^{2}\right)^{n+2}} d z d \bar{z}+c_{1}
\end{aligned}
$$

where $c_{1}, c_{2}$ etc ... will denote constants independent of $\varphi$. If we take polar coordinates $z=r e^{i \theta}$ and if we make the change of coordinates $r=e^{t / 2}, t \in \mathbb{R}$, we may write

$$
\begin{aligned}
\varphi(x) & =f(t), \\
\rho(t) & =\left(e^{t / 2}+e^{-t / 2}\right)^{-2}, \\
\rho_{i}(t) & =\frac{e^{i t}}{\left(1+e^{t}\right)^{n}} \rho(t),
\end{aligned}
$$

in which case

$$
\int_{X} \varphi d d^{c}(\varphi)=-\int_{-\infty}^{+\infty} \dot{f}(t)^{2} d t
$$

(where $\dot{f}(t)$ is the derivative of $f(t))$ and

$$
\int_{X} e^{\varphi} \frac{|z|^{2 i}}{\left(1+|z|^{2}\right)^{n+2}} d z d \bar{z}=2 \pi \int_{-\infty}^{+\infty} e^{f(t)} \rho_{i}(t) d t .
$$

We conclude that

$$
\begin{aligned}
A(\varphi) & =-\frac{1}{2} \int_{-\infty}^{+\infty} \dot{f}(t)^{2} d t \\
& +\sum_{i=0}^{n} \log \int_{-\infty}^{+\infty} e^{f(t)} \rho_{i}(t) d t+c_{2} .
\end{aligned}
$$

Furthermore

$$
\int_{X} \varphi \mu=\int_{-\infty}^{+\infty} f(t) \rho(t) d t=0
$$

4.3. Let $A>0$ be such that

$$
A^{2}=\int_{-\infty}^{\infty} \dot{f}(t)^{2} d t
$$


We first deduce from (25) that there is a constant $c_{3}$ such that

$$
|f(0)| \leq c_{3} A
$$

(compare [7] (8)). Indeed, the Cauchy-Schwarz inequality implies

$$
\left|\int_{s}^{t} \dot{f}(t) d t\right|^{2} \leq \int_{s}^{t} \dot{f}(t)^{2} d t \int_{s}^{t} d t
$$

i.e.

$$
(f(t)-f(s))^{2} \leq A^{2}|t-s|
$$

for all $s$ and $t$, hence

$$
-A \sqrt{|t-s|} \leq f(t)-f(s) \leq A \sqrt{|t-s|} .
$$

We multiply these inequalities by $\rho(s)$ and we integrate with respect to $s$. Since $\int_{-\infty}^{\infty} \rho(s) d s=1$ we get from $(25)$ that

$$
|f(t)| \leq A \int_{-\infty}^{+\infty} \sqrt{|t-s|} \rho(s) d s
$$

and (27) follows when $t=0$.

4.4. Lemma 2. There exists a function $u(t), t \in \mathbb{R}, t \geq 0$, which is $C^{1}$ and such that

i) $u(0)=f(0), u(+\infty)=f(+\infty)$;

ii) $\dot{u}(t) \geq 0, \dot{u}(t)$ is nonincreasing;

iii) $u(t) \geq f(t)$;

iv) $\int_{0}^{\infty} \dot{u}(t)^{2} d t=\int_{0}^{\infty} \dot{f}(t)^{2} d t$.

Proof of Lemma 2. Let $\dot{f}^{*}$ be the nonincreasing rearrangement of $\dot{f}$ on $[0,+\infty$ [ (cf. e.g. [8]). In other words $\dot{f}^{*}$ is the nonincreasing function on $[0,+\infty[$ such that, for all $y \geq 0,\left(\dot{f}^{*}\right)^{-1}(y)$ is the Lebesgue measure of the set of numbers $t \in[0,+\infty$ [ such that $\dot{f}(t) \geq y$. Since $\dot{f}$ is continuous, the same is true for $\dot{f}^{*}$ and we may define

$$
u(t)=f(0)+\int_{0}^{t} \dot{f}^{*}(s) d s .
$$


It is clear that $u(0)=f(0)$, and the standard equalities ([8], Lemma 2.2)

$$
\int_{0}^{+\infty}\left(\dot{f}^{*}\right)^{k}(s) d s=\int_{0}^{+\infty} \dot{f}^{k}(s) d s,
$$

for $k=1,2$, imply that $u(\infty)=f(\infty)$ and that iv) holds. Property ii) is a consequence of the definitions and iii) is equivalent to

$$
\int_{0}^{t} \dot{f}^{*}(s) d s \geq \int_{0}^{t} \dot{f}(s) d s,
$$

a well-known property of rearrangements.

q.e.d.

4.5. To bound the quantity $A(\varphi)$ in (24) we may now assume, by Lemma 2 , that $\dot{f}(t) \geq 0$, that $\dot{f}(t)$ is a nondecreasing function when $t \leq 0$ and a nonincreasing function when $t \geq 0$. Note that

$$
\log (a+b) \leq \log (2)+\log ^{+}(a)+\log ^{+}(b),
$$

where $\log ^{+}=\operatorname{Max}(\log , 0)$. Therefore, Lemma 3 below, when applied to $f(t)$ and $f(-t)$, gives

$$
\begin{aligned}
& \sum_{i=0}^{n} \log \int_{-\infty}^{+\infty} e^{f(t)} \rho_{i}(t) d t \\
\leq & \sum_{i=0}^{n} \log ^{+} \int_{0}^{+\infty} e^{f(t)} \rho_{i}(t) d t \\
+ & \sum_{i=0}^{n} \log ^{+} \int_{-\infty}^{0} e^{f(t)} \rho_{i}(t) d t+(n+1) \log (2) \\
\leq & \sum_{i=0}^{n} \log ^{+} \int_{0}^{+\infty} e^{f(t)} e^{-(i+1) t} d t \\
+ & \sum_{i=0}^{n} \log ^{+} \int_{-\infty}^{0} e^{f(t)} e^{(n-i-1) t} d t+(n+1) \log (2) \\
\leq & 2(n+1)|f(0)|+\left(\frac{1}{2}-\frac{1}{70 n^{2}}\right) A^{2}+c_{4} .
\end{aligned}
$$

From (27) we conclude that

$$
2(n+1)|f(0)| \leq 2(n+1) c_{3} A \leq \frac{1}{70 n^{2}} A^{2}+c_{5} .
$$


Therefore we get

$$
\sum_{i=0}^{n} \log \int_{-\infty}^{+\infty} e^{f(t)} \rho_{i}(t) \leq \frac{1}{2} A^{2}+c_{6}
$$

i.e. (by (24) and (26)) $A(\varphi)$ is bounded from above and Theorem 1 ii) is proved.

4.6. Lemma 3. Let $M \geq 1$ be an integer and let $u: \mathbb{R}^{+} \rightarrow \mathbb{R}$ be a $C^{1}$ map such that $\dot{u}$ is $L^{2}$ and nonincreasing. Define

$$
X=\sum_{j=0}^{M} \log \int_{0}^{+\infty} \exp (u(t)-(j+1) t) d t
$$

Then there exists a constant $C \geq 0$ such that

$$
X \leq(M+1)|u(0)|+\left(\frac{1}{2}-\frac{1}{70 M^{2}}\right) \int_{0}^{+\infty} \dot{u}(t)^{2} d t+C
$$

Proof of Lemma 3. For any integer $k \geq 1$ we let

$$
\lambda_{k}=1+\frac{1}{5 k^{2}}
$$

and

$$
\mu_{k}=1-\frac{1}{4 k}
$$

Note that $\lambda_{k} \cdot k+\mu_{k}>k$ and $r_{k}:=k+1-\lambda_{k} \cdot k-\mu_{k}=\frac{1}{4 k}-\frac{1}{5 k}>0$.

Define $N \geq 0$ as the smallest integer such that

$$
\dot{u}(0) \leq \lambda_{N+1} \cdot(N+1)+\mu_{N+1}
$$

* If $j \geq N+1$ and $t \geq 0$ we have

$$
\dot{u}(t)-(j+1) \leq \dot{u}(0)-(j+1) \leq \dot{u}(0)-(N+2) \leq-r_{N+1} .
$$

Therefore

$$
\log \int_{0}^{\infty} \exp (u(t)-(j+1) t) d t \leq u(0)+\log \int_{0}^{\infty} \exp \left(-r_{N+1} t\right) d t=u(0)+c
$$


* If $N=0$ and $0 \leq j<N+1$, i.e. $j=0$, we know from (28) that

$$
u(t) \leq u(0)+\sqrt{I \cdot t}
$$

where

$$
I=\int_{0}^{\infty} \dot{u}(t)^{2} d t .
$$

Therefore, by completing a square we get

$$
u(t)-t \leq u(0)+\sqrt{I \cdot t}-t \leq u(0)+\frac{3 I}{8}-\frac{t}{3},
$$

from which it follows that

$$
\log \int_{0}^{\infty} \exp (u(t)-t) d t \leq u(0)+\frac{3 I}{8}+\log \int_{0}^{\infty} \exp (-t / 3) d t
$$

hence, if $N=0$,

$$
X \leq(M+1) u(0)+\frac{3 I}{8}+C .
$$

$*$ If $N \geq 1$, we let $\lambda=\lambda_{N}, \mu=\mu_{N}$ and we choose real numbers $x_{0} \geq x_{1} \geq \cdots \geq$ $x_{N}>0$ such that

$$
\dot{u}\left(x_{j}\right)=\lambda \cdot j+\mu, \quad 0 \leq j<N .
$$

Then if $0 \leq j<N$, we have

$$
\begin{gathered}
u(t)-(j+1) t=u(t)-(\lambda \cdot j+\mu) t+(\lambda \cdot j+\mu-(j+1)) t \\
\leq u\left(x_{j}\right)-(\lambda \cdot j+\mu) x_{j}+(\lambda \cdot N+\mu-(N+1)) t
\end{gathered}
$$

hence

$$
\begin{aligned}
& \log \int_{0}^{\infty} \exp (u(t)-(j+1) t) d t \leq \\
& u\left(x_{j}\right)-(\lambda \cdot j+\mu) x_{j}+\log \int_{0}^{\infty} \exp \left(-r_{N} t\right) d t \\
&=\varphi\left(x_{j}\right)+c^{\prime},
\end{aligned}
$$

where

$$
\varphi(x)=u(x)-\dot{u}(x) x .
$$

Therefore

$$
X \leq(M-N)(u(0)+c)+N c^{\prime}+Y
$$


where

$$
Y=\sum_{j=0}^{N} \varphi\left(x_{j}\right) .
$$

Using (31) we can write

$$
\begin{aligned}
Y & =\sum_{j=0}^{N} \varphi\left(x_{j}\right)=\frac{1}{\lambda} \sum_{j=1}^{N} \varphi\left(x_{j}\right)\left(\dot{u}\left(x_{j}\right)-\dot{u}\left(x_{j-1}\right)\right)+\varphi\left(x_{0}\right) \\
& =\frac{1}{\lambda} \sum_{j=1}^{N-1} \dot{u}\left(x_{j}\right)\left(\varphi\left(x_{j}\right)-\varphi\left(x_{j+1}\right)\right)-\frac{1}{\lambda} \varphi\left(x_{1}\right) \dot{u}\left(x_{0}\right) \\
& +\frac{1}{\lambda} \dot{u}\left(x_{N}\right) \varphi\left(x_{N}\right)+\varphi\left(x_{0}\right) \\
& =\frac{1}{\lambda} \sum_{j=0}^{N-1} \dot{u}\left(x_{j}\right)\left(\varphi\left(x_{j}\right)-\varphi\left(x_{j+1}\right)\right)+\frac{1}{\lambda} \dot{u}\left(x_{N}\right)\left(\varphi\left(x_{N}\right)-\varphi(0)\right) \\
& +\left(N+\frac{\mu}{\lambda}\right) u(0)+\varphi\left(x_{0}\right)\left(1-\frac{\dot{u}\left(x_{0}\right)}{\lambda}\right) \\
& \leq \frac{1}{\lambda} \int_{0}^{x_{0}} \dot{u} \dot{\varphi} d t+\left(N+\frac{\mu}{\lambda}\right) u(0)+\varphi\left(x_{0}\right)\left(1-\frac{\dot{u}\left(x_{0}\right)}{\lambda}\right) .
\end{aligned}
$$

But

$$
\begin{aligned}
\int_{0}^{x_{0}} \dot{u} \dot{\varphi} d t & =-\int_{0}^{x_{0}} t \dot{u} \ddot{u} d t \\
& =\left[-\frac{1}{2} t \dot{u} \dot{u}\right]_{0}^{x_{0}}+\frac{1}{2} \int_{0}^{x_{0}} \dot{u}(t)^{2} d t \\
& =-\frac{1}{2} \dot{u}\left(x_{0}\right)^{2} x_{0}+\frac{1}{2} \int_{0}^{x_{0}} \dot{u}(t)^{2} d t
\end{aligned}
$$

So we get

$$
Y \leq \frac{1}{2 \lambda} I+\left(N+\frac{\mu}{\lambda}\right) u(0)+R
$$

where

$$
\begin{aligned}
R & =-\frac{1}{2 \lambda} \dot{u}\left(x_{0}\right)^{2} x_{0}+\varphi\left(x_{0}\right)\left(1-\frac{\dot{u}\left(x_{0}\right)}{\lambda}\right) \\
& =\left(1-\frac{\mu}{\lambda}\right) u\left(x_{0}\right)+\left(\frac{\mu^{2}}{2 \lambda}-\mu\right) x_{0} .
\end{aligned}
$$

Now, by (28),

$$
u\left(x_{0}\right) \leq u(0)+\sqrt{x_{0} \cdot I},
$$


and, by completing the square,

$$
\alpha \sqrt{x_{0}}-\beta x_{0} \leq \frac{\alpha^{2}}{4 \beta} \quad \text { for all } \alpha, \beta>0 .
$$

Therefore

$$
R \leq\left(1-\frac{\mu}{\lambda}\right) u(0)+\frac{(1-\mu / \lambda)^{2}}{4\left(\mu-\frac{\mu^{2}}{2 \lambda}\right)} I .
$$

Using (32) we get

$$
Y \leq(N+1) u(0)+A \cdot I
$$

where

$$
A=\frac{1}{2 \lambda}+\frac{\left(1-\frac{\mu}{\lambda}\right)^{2}}{4\left(\mu-\frac{\mu^{2}}{2 \lambda}\right)} .
$$

From the values of $\lambda=\lambda_{N}$ and $\mu=\mu_{N}$ we compute

$$
A \leq \frac{1}{2}-\frac{1}{70 N^{2}} \leq \frac{1}{2}-\frac{1}{70 M^{2}}
$$

Therefore

$$
X \leq(M+1) u(0)+\left(\frac{1}{2}-\frac{1}{70 M^{2}}\right) \int_{0}^{\infty} \dot{u}(t)^{2} d t+C
$$

q.e.d.

\section{Flat bundles}

According to Bismut and Zhang [2] a flat $C^{\infty}$ bundle $(E, \nabla), \nabla^{2}=0$, together with a $C^{\infty}$ metric $h$ on $E$ on a $C^{\infty}$ manifold $M$ is the analog in the differentiable category of a hermitian holomorphic bundle on a complex manifold. Inequalities similar to our conjecture might also hold in this case, but in some cases they must be lower bounds rather than upper bounds, as the following example suggests.

Let $M=S^{1}$ be the circle, and let $E=\mathbb{C}$ be the trivial line bundle on $M$. We equip $M$ with its standard metric and $E$ with an arbitrary metric $h$. The connection $\nabla=d$ has an adjoint $d^{*}$ (depending on $h$ ), and we consider the Laplace operator $\Delta=d^{*} d$ on $C^{\infty}(M)$, and its regularized determinant $\operatorname{det}^{\prime}(\Delta)$.

Proposition 3. There is a constant $C(E)$ such that, for any choice of a metric $h$ on $E=\mathbb{C}$, the following inequality holds

$$
\operatorname{det}^{\prime}(\Delta) \leq C(E) \text {. }
$$


Proof. We have $H^{0}\left(S^{1}, \mathbb{C}\right) \simeq H^{1}\left(S^{1}, \mathbb{C}\right) \simeq \mathbb{C}$. On $\lambda(E)=H^{0}\left(S^{1}, \mathbb{C}\right) \otimes H^{1}\left(S^{1}, \mathbb{C}\right)^{*}$ we define the Quillen metric by

$$
\|\cdot\|_{Q}^{2}=\|\cdot\|_{L_{2}}^{2} \operatorname{det}^{\prime}(\Delta)^{-1}
$$

as in $\S 1$ above [2].

If $\varphi \in C^{\infty}(M)$ we let $h$ be the metric on $E=\mathbb{C}$ such that $h(1,1)=\exp (\varphi)$, and we denote by $\Delta_{\varphi}$ the corresponding Laplace operator on $C^{\infty}(M, \mathbb{C})$. We define

$$
\begin{aligned}
A(\varphi) & =\log \left(\operatorname{det}^{\prime}\left(\Delta_{\varphi}\right)\right)-\log \left(\operatorname{det}^{\prime}\left(\Delta_{0}\right)\right) \\
& =\log \frac{\|\cdot\|_{L^{2}, \varphi}^{2}}{\|\cdot\|_{L^{2}, 0}^{2}}-\log \frac{\|\cdot\|_{Q, \varphi}^{2}}{\|\cdot\|_{Q, 0}^{2}} .
\end{aligned}
$$

According to [2] Theorem 0.1 we have

$$
\log \frac{\|\cdot\|_{Q, \varphi}}{\|\cdot\|_{Q, 0}}=0
$$

and a computation similar to $\S 2.3$ gives

$$
\log \frac{\|\cdot\|_{L^{2}, \varphi}^{2}}{\|\cdot\|_{L^{2}, 0}^{2}}=\log \int_{S^{1}} e^{\varphi(x)} d x+\log \int_{S^{1}} e^{-\varphi(x)} d x,
$$

where $d x$ is the Haar measure of length one. The Cauchy-Schwarz inequality implies

$$
\left(\int_{S^{1}} d x\right)^{2} \leq\left(\int_{S_{1}} e^{\varphi(x)} d x\right)\left(\int_{S^{1}} e^{-\varphi(x)} d x\right)
$$

from which we conclude that $A(\varphi) \geq 0$.

q.e.d.

\section{References.}

[1] Bismut J.-M., Gillet H., Soulé C.: Analytic torsion and holomorphic determinant bundles I. Bott-Chern forms and analytic torsion, Comm. in Math. Physics 115 (1988), 49-78.

[2] Bismut J.-M., Zhang W.: An extension of the Cheeger-Muller theorem, Astérisque 205 (1992), Soc. Math. de France, Paris. 
[3] Fontana L.: Sharp borderline Sobolev inequalities on compact Riemannian manifolds, Comm. Math. Helv. 68 (1993), 415-454.

[4] Gillet H., Soulé C.: Characteristic classes for algebraic vector bundles with hermitian metrics I, Annals of Math. 131 (1990), 163-203.

[5] Gillet H., Soulé C.: An arithmetic Riemann-Roch theorem, Inventiones Math. 110 (1992), 473-543.

[6] Kiessling M.K.H.: Statistical mechanics of classical particles with logarithmic interactions, Comm. Pure Appl. Math. 46 (1993), 27-56.

[7] Moser J.: A sharp form of an inequality by N. Trudinger, Indiana Math. J. 20 (1971), 1077-1092.

[8] O'Neil R.: Convolution operators and $L(p, q)$ spaces, Duke Math. J. 30 (1963), 129-142.

[9] Quillen D.: Determinants of Cauchy-Riemann operators over a Riemann surface, Funct. Anal. Appl. (1985), 31-34.

[10] Soulé C.: A vanishing theorem on arithmetic surfaces, Inventiones Math. 116 (1994), 577-599.

H.G.: Department of Mathematics, Statistics, and Computer Science, University of Illinois at Chicago, 851 S. Morgan Street, Chicago, IL 60607-7045, U.S.A.

C.S. : CNRS, Institut des Hautes Études Scientifiques, 35, Route de Chartres, 91440, Bures-sur-Yvette, France 